\title{
The Autonomous Sensory Meridian Response Activates the Parasympathetic Nervous System
}

\section{Kanae Tada}

Chukyo University

\section{Takahiro Ezaki}

The University of Tokyo

Hirohito M. Kondo ( $\nabla$ kondo@lets.chukyo-u.ac.jp )

Chukyo University

\section{Research Article}

Keywords: ASMR, hearing, multisensory, interoception, PPG

Posted Date: November 4th, 2021

DOI: https://doi.org/10.21203/rs.3.rs-1026254/v1

License: (1) This work is licensed under a Creative Commons Attribution 4.0 International License. Read Full License 


\section{Abstract}

The autonomous sensory meridian response (ASMR) produces a tingling sensation with positive feelings when binaural sounds are presented through headphones. This crossmodal response is believed to be related to sensory integration, but its psychological and physiological mechanisms are largely unknown. We conducted two experiments (on thirty and forty-two participants, respectively) to measure the subjective intensity of ASMR, finger photoplethysmography (PPG), and shift in mental states during their ASMR experience. We found that the intensity of ASMR was greater when auditory and visual stimuli were presented simultaneously than when only auditory stimuli were presented. This suggests the involvement of sensory-based, bottom-up processing. Furthermore, their pulse rates decreased and PPG amplitudes increased during the ASMR experience. Self-reported measures showed that the ASMR experience shifted participant's negative moods to neutral mental states. These results suggest that the parasympathetic nervous system is dominant during the ASMR experience.

\section{Introduction}

Auditory events occurring in close proximity to the head can evoke skin-related sensations. We may experience a tingling sensation across the scalp and back of the neck, termed the autonomous sensory meridian response (ASMR), while listening to binaural sounds with headphones ${ }^{1}$. Such cross-modal bodily sensations provide clues to investigate underlying interaction between the auditory and somatosensory systems. However, it is unclear how auditory inputs shape body schema.

ASMR seems to differ from music-induced chills, often characterized as a subjective response accompanied by goose bumps and strong emotions. From the psychological perspective, the ASMR can be induced by various types of stimuli and affected by sound localization ${ }^{1,2}$, whereas musical chills strongly depend on sound properties ${ }^{3-5}$. ASMR stimuli frequently induce a feeling of relaxation and aid sleep ${ }^{1,6}$, whereas musical chills are associated with a profound emotional experience and shivers ${ }^{7,8}$. From the physiological perspective, experience of musical chills is often accompanied with goose bumps on the arms ${ }^{9}$, which suggests the involvement of the sympathetic nervous system ${ }^{10}$. However, it is unresolved whether the ASMR induces substantial changes in physiological states. We show that the ASMR activates the parasympathetic nervous system.

We conducted two experiments to clarify the psychological and physiological characteristics of the ASMR experience. In Experiment 1, we investigated whether sound-induced ASMR is enhanced or reduced by simultaneous presentation of visual stimuli (Figure 1A). Bodily sensations can be modified by the integration of multisensory information ${ }^{11}$. In the rubber-hand illusion, observers watch a rubber hand being stroked synchronously with their own hand and fail to localize the perceived position of their hand 12. The spatial resolution of passive touch is better when the arm is visible than when it is not ${ }^{13}$. These findings indicate a close relationship between visual and tactile modalities. Given that the ASMR is derived from bottom-up processing, bodily sensations could be increased with the addition of visual information. It is also known that skin-related modalities are influenced by one's attention and 
expectation ${ }^{14,15}$. Thus, it is possible that participant curiosity about invisible sound sources shapes the ASMR. In such a case, the subjective intensity of the ASMR could be reduced by the prior knowledge of sound sources (i.e., visual information). We tested these hypotheses in Experiment 1.

We used finger photoplethysmography (PPG) to examine physiological responses during the ASMR in Experiment 2 (Figure 1B). Several studies have demonstrated that musical chills are associated with increases in electrodermal activity, pupil diameter, or heart rate ${ }^{8,16}$. These findings indicate that the sympathetic nervous system is dominant during musical chills. A recent study using ASMR stimuli showed that the skin conductance level increases and heart rate decreases while participants watch ASMR videos ${ }^{17}$. This means that the ASMR is associated with activation and deactivation of the autonomic nervous system. As described above, ASMR users frequently want to experience a feeling of relaxation, rather than excitement. Given that the ASMR activates the parasympathetic nervous system, it is possible that, due to blood vessel dilation, the pulse wave amplitude increases; due to slowing of the heart, pulse rate decreases. Using the Profile of Mood States 2nd Edition (POMS 2), we checked participant mood states before and after they experienced ASMR stimuli. We examined transformation processes from sensory inputs to bodily sensations by means of changes in ASMR estimates, physiological responses, and mood states.

\section{Methods}

\section{Participants}

Thirty-two college students were recruited for Experiment 1. Two of the participants were excluded from subsequent analyses because they did not perceive tingling nor tickling sensations, leaving data from 30 participants (12 males and 18 females; mean \pm SD age $=20.5 \pm 0.13$ years, range 19-

26). Participants were randomly assigned to either the audiovisual or audio-only condition. Numbers of males and females were counterbalanced between the two conditions to control for gender effects. Another group of 44 college students participated in Experiment 2. Data from two participants were lost because of technical problems, leaving data from 42 participants ( 14 males and 28 females; mean \pm SD age $=21.9 \pm 2.3$ years, range $20-33$ ). For each experiment, 12 and 30 participants reported that they regularly use ASMR media, but they had never watched the ASMR stimuli used in this study. According to a priori power analyses with a power of $0.8(a$-level $=0.05)$, we required 32 participants for Experiment 1 to detect a main effect (effect size $=0.4$; mixed-design analysis of variance: ANOVA) and 46 participants for Experiment 2 to detect significant correlations ( $r=0.4$; bivariate normal model). This study was approved by the Research Ethics Committee of Chukyo University (approval no. RS19-015) and carried out in accordance with the Ethical Guidelines for Medical and Health Research Involving Human Subjects. All participants were right-handed with normal hearing and with normal or corrected-to-normal vision. They gave written informed consent after the procedures had been fully explained to them.

\section{Stimuli and procedures}


Experiment 1 consisted of audiovisual and audio-only conditions, including five 2-min trials for each (Figure 1A). ASMR videos with Creative Commons licenses were taken from YouTube (https://www.youtube.com/watch?v=7MZtaAgqoTY and https://www.youtube.com/watch? $v=a s G L p 12 N S I E \& t=2031 s$ ). Audiovisual stimuli were edited from segments of the videos that gave tingling sensations to all of the authors. There was no facial information in the stimuli to exclude confounding effects of facial expressions on participant responses. Participants could explicitly observe sound sources of the stimuli under the audiovisual condition. For the audio-only condition, visual information was removed from the stimuli. Participants were instructed to continuously indicate their tingling sensation using a 4-point Likert scale: "no ASMR", "weak ASMR", "moderate ASMR", and "strong ASMR." Stimulus presentation and data collection were controlled using a PC with Presentation software (Neurobehavioral Systems, Berkeley, CA, USA). Visual stimuli were presented on an LCD monitor with a temporal resolution of $60 \mathrm{~Hz}$. Participants viewed the stimuli with a spatial resolution of $1,280 \times 720$ pixels (visual angle, $16.0 \times 9.0$ degree) at a distance of approximately $57 \mathrm{~cm}$. They dichotically listened to the stimuli through Sennheiser HD 599 headphones. Responses of a participant's right hand were collected via four keys on a computer keyboard with a sampling rate of $1,000 \mathrm{~Hz}$. A key press indicating a response was held until a subsequent key press. In interviews conducted after Experiment 1, participants reported the degree of tingling and tickling sensations throughout the experiment using a visual analogue scale (range $0-100)$.

In Experiment 2, the test period consisting of three 2-min trials was placed between 2-min rest trials (Figure 1B). There was no gap between the trials. Participants experienced audiovisual stimuli while PPG signals were recorded using MP36 data acquisition units (Biopac Systems, Goleta, CA, USA) with the SS4LA PPG transducer. The stimuli used in trials 2, 3, and 4 in Experiment 1 were presented during the test period. The task procedure was the same as that under the audiovisual condition of Experiment 1. A PPG sensor was attached to a participant's left middle finger. PPG data were recorded at a sampling rate of 2,000 Hz using the software Biopac Student Lab Pro (version 4.1). Participants were instructed to minimize their body movements throughout the 10-min data-recording period.

Before and after Experiment 2, we obtained self-reported measures of participant mood states using the POMS $2^{38}$. The POMS 2 reflects an individual's current mood and comprises six subscales: AngerHostility (AH), Confusion-Bewilderment (CB), Depression-Dejection (DD), Fatigue-Inertia (FI), TensionAnxiety (TA), and Vigor-Activity (VA). The POMS 2 also includes a summary scale called a Total Mood Disturbance score and a Friendliness (F) subscale for testing positive mood in a complementary fashion. Participants filled out a questionnaire in a quiet room.

\section{Data analyses}

For behavioral data, participant responses were converted to an ASMR estimate $(0,1,2$, or 3$)$. The intensity of the ASMR for each participant was computed by averaging the ASMR estimate over the test period (1-ms bins). The intensity of tingling and tickling sensations throughout the experiment was 
derived from the data of a visual analogue scale. The Total Mood Disturbance score for the POMS 2 was calculated by subtracting the VA subscore from the $\mathrm{AH}, \mathrm{CB}, \mathrm{DD}, \mathrm{FI}$, and TA subscores. We used the Bonferroni correction for post-hoc comparisons ( $a$-level $=0.05)$ when conducting ANOVA. Statistical analyses were carried out with IBM SPSS Statistics (ver. 25).

PPG data were preprocessed using high- and low-pass filtering at $0.5-$ and $35-\mathrm{Hz}$ cutoff frequencies. Using the preprocessed data, pulse rate was evaluated for each participant. We extracted time points of peaks for all spikes and computed peak-to-peak intervals (PPIs). PPI values that deviated more than 3 SDs from the mean were removed as outliers in subsequent analyses. The pulse rate was calculated as the inverse of the PPI. The SD of the pulse rate was used as a measure of pulse rate variability. We also computed the pulse wave amplitude as the difference between the maximum and minimum values of the PPG signal in each spike interval.

\section{Results}

\section{Experiment 1: Effects of auditory and visual inputs on ASMR}

Figure 2A shows time-series data of the ASMR estimate. We conducted a mixed-design 2 (condition types) $\times 5$ (trial types) ANOVA on the ASMR estimate (Figure 2B). The ASMR estimate (mean \pm standard error) was greater for audiovisual conditions $(1.36 \pm 0.13)$ than for audio-only conditions $(0.96 \pm$ 0.13): $F(1,28)=5.12, p=0.032, \eta_{\mathrm{p}}^{2}=0.16$. ASMR estimates were $0.96 \pm 0.09$ for trial $1,1.21 \pm 0.08$ for trial $2,1.52 \pm 0.13$ for trial $3,1.00 \pm 0.10$ for trial 4 , and $1.12 \pm 0.13$ for trial 5 . The main effect of the trial type was significant: $F(4,112)=10.42, p<0.001, \eta_{\mathfrak{p}}^{2}=0.27$. However, the interaction between condition and trial types was not significant: $F(4,112)=1.70, p=0.15, \quad \eta_{\mathrm{p}}^{2} \quad=0.06$. These results suggest that sound-induced ASMR is generally enhanced by the simultaneous presentation of visual stimuli, regardless of the stimuli.

We evaluated tingling and tickling sensations throughout the experiment (Figure 2C). The intensity of tingling and tickling sensations was greater under audiovisual conditions than under audio-only conditions: $69.0 \pm 2.2$ and $49.9 \pm 6.6$ for tingling, $t=2.76, p=0.010$, Cohen's $d=1.01 ; 61.7 \pm 5.7$ and 42.1 \pm 7.6 for tickling, $t=2.05, p=0.050$, Cohen's $d=0.75$. We examined the degree to which these measures were associated with the averaged ASMR estimate for each participant (Figure 2D). The ASMR estimate was positively correlated with the intensity of tingling and tickling sensations: $r=0.57, p=0.001 ; r=0.58$, $p=0.001$. Consistent with previous findings, the pattern of the correlations indicates that the ASMR experience is accompanied with tingling and tickling sensations.

\section{Experiment 2: ASMR and PPG}


Thus far, we have demonstrated additive effects of multisensory inputs on ASMR estimates. We chose the audiovisual stimuli from the ASMR videos used in Experiment 1. PPG and ASMR results are shown in Figure 3A. Pulse wave amplitude increased during test periods, compared with rest periods: $F(4,164)=$ $6.89, p<0.001, \eta_{\mathfrak{p}}^{2}=0.14$. In contrast, the pulse rate decreased during test periods, compared with rest periods: $F(4,164)=14.20, p<0.001, \eta_{\mathfrak{p}}^{2}=0.26$. The pattern of the results indicates that the ASMR experience is associated with dominance of the parasympathetic nervous system.

We focused on intra-individual variability of the measures (Figure 3B). Pulse wave amplitude was larger during the test period $(0.554 \pm 0.043)$ than during the rest period $(0.495 \pm 0.038): t(41)=3.68, p=$ 0.001 , Cohen's $d=0.22$. Pulse rate was lower during the test period $(78.3 \pm 1.6 \mathrm{bpm})$ than during the rest period $(80.6 \pm 1.6 \mathrm{bpm}): t(41)=4.27, p<0.001$, Cohen's $d=0.22$. Furthermore, variability of pulse wave amplitude was smaller and pulse rate was reduced during the test period $(0.086 \pm 0.009$ and $4.6 \pm 0.2$ bpm, respectively) compared to the rest period $(0.107 \pm 0.010$ and $5.5 \pm 0.3 \mathrm{bpm}$, respectively): $t(41)=$ $3.57, p=0.001$, Cohen's $d=0.34 ; t(41)=5.23, p<0.001$, Cohen's $d=0.73$. It is possible that the reduction of intra-individual variability in both amplitude- and time-domains indicates participant relaxation.

We investigated whether these measures during the test period were associated with the ASMR estimate (Figure 3C). The ASMR estimate showed a significant correlation with the pulse rate $(r=-0.49, p=0.001)$, but not with the pulse wave amplitude $(r=0.16, p=0.30)$. The correlations between the ASMR estimate and variability measures did not reach statistical significance: $|r|<0.06$. Thus, pulse rate is more reliable for predicting subjective intensity of the ASMR experience than pulse wave amplitude.

\section{Experiment 2: ASMR and mood states}

We investigated effects of the ASMR experience on changes in participant mood states. The Total Mood Disturbance score decreased after the experiment $(25.1 \pm 4.1)$ compared to before the experiment $(38.7 \pm$ $5.1): t(41)=5.08, p<0.001$, Cohen's $d=0.46$. All subscores after the experiment were lower than those before the experiment (Figure 4). Specifically, the differences in the CB, DD, and TA subscores reached statistical significance: $t(41)>2.79, p<0.008$, Cohen's $d>0.27$. The reduction of negative moods reflects a bias that the ASMR experience moves participant moods toward neutral mental states.

\section{Discussion}

Behavioral results demonstrated that the ASMR estimate was greater under audiovisual conditions than under audio-only conditions. This additive effect of visual information suggests that the ASMR is largely affected by sensory-based, bottom-up processing. The ASMR estimate was associated with enhancement in pulse wave amplitude and with reduction of the pulse rate. The physiological results indicate a parasympathetic shift. Self-reported measures showed that the ASMR experience moved 
participants from negative moods to neutral mental states. Therefore, we conclude that the ASMR activates the parasympathetic nervous system.

We revealed that multisensory (i.e., visual and auditory) inputs strengthened the ASMR, accompanied with tingling and tickling sensations. The ASMR is similar to the rubber-hand and body-swap illusions because sensory inputs influence bodily awareness and sensations. In these illusions, the ownership of an artificial hand and body can be induced by tactile stimulation on the hand or body and its visual information ${ }^{12,18}$. Several researchers have pointed out that the rubber-hand illusion is based on Bayesian perceptual learning ${ }^{19,20}$. The sensitivity to ASMR stimuli, as well as to the rubber-hand illusion, varies widely among individuals, but is preserved within individuals ${ }^{2}$. Thus, an individual's preference for ASMR videos probably represents a convergence of accumulating experience of various videos and sharpening the positive emotional aspects of ASMR videos.

Multisensory interactions frequently occur in the space surrounding one's body 21,22 because such a mechanism is critical to grasp the peripersonal space as a point of contact with the environment ${ }^{23}$. A tingling sensation was enhanced by sounds moving around the head ${ }^{24}$. Also, a strong tickling sensation occurred when the sound of an ear being stroked was presented with headphones ${ }^{25}$. Intriguingly, there is a case in which external stimulation is not a prerequisite for tingling sensation ${ }^{15}$. Focusing one's attention on a body part can give rise to various spontaneous sensations, including tingling 26,27 . A spontaneous tingling sensation more frequently occurred on fingers and toes than on the trunk ${ }^{28}$. The most intense sensations were reported at the tips of the fingers ${ }^{26}$. These results are probably related to the gradient of peripheral receptor density. Several researchers have argued that left-hand dominance of the spontaneous tingling sensation indicates right-hemisphere central processes ${ }^{26,27}$. In contrast, the ASMR mainly originates on the scalp and back of the neck, and laterality of body parts does not occur ${ }^{1,2}$. Thus, the ASMR differs from a spontaneous sensation triggered by endogenous attention.

The pulse wave amplitude increased and pulse rate decreased during the ASMR experience, indicating the dominance of the parasympathetic nervous system. This is consistent with previous findings showing that the ASMR experience leads to a reduction of participant negative moods, including depression and anxiety ${ }^{1}$. A previous study demonstrated that the heart rate decreased for ASMR responders, but did not for non-responders, when participants watched ASMR videos ${ }^{17}$. Physiological responses observed in this study were not derived from the effect of anticipation in each individual because none had watched our audiovisual stimuli previously. In addition, we demonstrated that the pulse rate was a critical index to predict the subjective intensity of the ASMR. Therefore, our results provide general principles of physiological responses during the ASMR experience.

We presented empirical evidence supporting the report that Internet users frequently want ASMR videos to relax and calm down. In previous studies, $82 \%$ of respondents agreed that they used ASMR videos to aid sleep ${ }^{1}$ and $63 \%$ of respondents answered that they watched such videos before sleeping ${ }^{29}$. Anecdotal reports about introducing sleep are consistent with our physiological findings that the ASMR calms the 
body. Specifically, we showed that an individual's ASMR intensity was closely linked to his/her pulse rate. Social isolation under the COVID-19 pandemic has led to an increase of mental illnesses, such as stress, anxiety, and depression ${ }^{30}$. Thus, the pulse rate can be a useful biomarker for assessment of psychological health, because PPG sensors are commonly implemented in wearable watch devices.

Several studies of emotion have used music to clarify neural correlates of chills, arguing that musical chills are closely linked to activation of paralimbic areas for emotion and reward processing, rather than those for auditory processing itself 31,32 . Heart rate increases during musical chills ${ }^{8,31,33}$. Chills are known as a phenomenon in which auditory inputs frequently cause goose bumps on the arms 7,9 , whereas only $15 \%$ of ASMR responders reported tingling sensation on their arms ${ }^{2}$. Although the ASMR and musical chills accompany pleasant emotions, the balance of autonomic functions could account for the different physiological responses of these phenomena. Even though misophonia induces negative emotional reactions to particular sounds (i.e., the opposite of the ASMR), several researchers have noted the similar sensitivity between the ASMR and misophonia ${ }^{1,29}$. However, misophonic triggers seem to increase the heart rate ${ }^{34}$. Hence, given that we specify acoustic features affecting the ASMR, chills, and misophonia, it should be possible to design comfortable sound landscapes to improve the quality of life 35 .

We showed that the ASMR experience relies on bottom-up processing, induced parasympathetic shift, and reduced negative moods. Investigating the ASMR can initiate not only new multisensory research, but also clinical investigation into treatments for depressive moods and mental stress. It has been argued that chills reflect threatening situations ${ }^{36}$ or aesthetic awe ${ }^{37}$. Poerio et al. (2018) noted the possibility that the ASMR simulates some sort of social grooming. Thus, considering the biological validity of the ASMR will be important in future studies.

\section{Declarations}

\section{Acknowledgments}

This study was supported by JSPS KAKENHI grant (no. $20 \mathrm{H} 01789$ to TE and HMK). We thank Hsin-I Liao for her comments on the research design and Manami Ueda for her help in data collection.

\section{Authors' Contributions}

Conceptualization: KT and HMK; Data curation: KT, TE, and HMK; Formal analysis: KT, TE, and HMK; Funding acquisition: TE and HMK; Investigation: KT and HMK; Methodology: KT, TE, and HMK; Project administration: HMK; Resources: TE and HMK; Supervision: HMK; Writing - original draft: $K T$, TE, and HMK. 


\section{Competing Interests}

We declare that we have no competing interests.

\section{References}

1 Barratt, E. L. \& Davis, N. J. Autonomous Sensory Meridian Response (ASMR): a flow-like mental state. Peer 3, e851, doi:10.7717/peerj.851 (2015).

2 Koumura, T., Nakatani, M., Liao, H.-I. \& Kondo, H. M. Dark, loud, and compact sounds induce frisson. Q. J. Exp. Psychol.74, 1140-1152, doi:10.1177/1747021820977174 (2021).

3 Goldstein, A. Thrills in response to music and other stimuli. Physiological Psychology8, 126-129, doi:10.3758/BF03326460 (1980).

4 Panksepp, J. The emotional sources of "chills" induced by music. Music Percept.13, 171-207, doi:10.2307/40285693 (1995).

5 Grewe, O., Kopiez, R. \& Altenmüller, E. Chills as an indicator of individual emotional peaks. Ann. N. Y. Acad. Sci.1169, 351-354, doi:10.1111/j.1749-6632.2009.04783.x (2009).

6 Barratt, E. L., Spence, C. \& Davis, N. J. Sensory determinants of the autonomous sensory meridian response (ASMR): understanding the triggers. PeerJ5, e3846, doi:10.7717/peerj.3846 (2017).

7 Harrison, L. \& Loui, P. Thrills, chills, frissons, and skin orgasms: toward an integrative model of transcendent psychophysiological experiences in music. Front. Psychol.5, 790, doi:10.3389/fpsyg.2014.00790 (2014).

8 Mori, K. \& Iwanaga, M. Two types of peak emotional responses to music: the psychophysiology of chills and tears. Scientific Reports7, 46063, doi:10.1038/srep46063 (2017).

9 Craig, D. G. An exploratory study of physiological changes during "chills" induced by music. Musicae Scientiae9, 273-287, doi:10.1177/102986490500900207 (2005).

10 Lindsley, D. B. \& Sassaman, W. H. Autonomic activity and brain potentials associated with "voluntary" control of the pilomotors (mm. Arrectores pilorum). J. Neurophysiol.1, 342-349, doi:10.1152/jn.1938.1.4.342 (1938).

11 Maravita, A., Spence, C. \& Driver, J. Multisensory integration and the body schema: close to hand and within reach. Curr. Biol.13, R531-R539, doi:10.1016/s0960-9822(03)00449-4 (2003).

12 Botvinick, M. \& Cohen, J. Rubber hands 'feel' touch that eyes see. Nature391, 756, doi:10.1038/35784 (1998). 
13 Kennett, S., Taylor-Clarke, M. \& Haggard, P. Noninformative vision improves the spatial resolution of touch in humans. Curr. Biol.11, 1188-1191, doi:10.1016/s0960-9822(01)00327-x (2001).

14 Jensen, K. B. et al. Nonconscious activation of placebo and nocebo pain responses. Proc. Natl. Acad. Sci. U. S. A.109, 15959-15964, doi:10.1073/pnas.1202056109 (2012).

15 Tihanyi, B. T., Ferentzi, E., Beissner, F. \& Köteles, F. The neuropsychophysiology of tingling. Conscious. Cogn.58, 97-110, doi:10.1016/j.concog.2017.10.015 (2018).

16 Laeng, B., Eidet, L. M., Sulutvedt, U. \& Panksepp, J. Music chills: the eye pupil as a mirror to music's soul. Conscious. Cogn.44, 161-178, doi:10.1016/j.concog.2016.07.009 (2016).

17 Poerio, G. L., Blakey, E., Hostler, T. J. \& Veltri, T. More than a feeling: autonomous sensory meridian response (ASMR) is characterized by reliable changes in affect and physiology. PLoS ONE13, e0196645, doi:10.1371/journal.pone.0196645 (2018).

18 Petkova, V. I. \& Ehrsson, H. H. If I were you: perceptual illusion of body swapping. PLoS ONE3, e3832, doi:10.1371/journal.pone.0003832 (2008).

19 Armel, K. C. \& Ramachandran, V. S. Projecting sensations to external objects: evidence from skin conductance response. Proc. R. Soc. B.270, 1499-1506, doi:10.1098/rspb.2003.2364 (2003).

20 Tsakiris, M. \& Haggard, P. The rubber hand illusion revisited: visuotactile integration and selfattribution. J. Exp. Psychol. Hum. Percept. Perform.31, 80-91, doi:10.1037/0096-1523.31.1.80 (2005).

21 Occelli, V., Spence, C. \& Zampini, M. Audiotactile interactions in front and rear space. Neurosci. Biobehav. Rev.35, 589-598, doi:10.1016/j.neubiorev.2010.07.004 (2011).

22 Zampini, M., Torresan, D., Spence, C. \& Murray, M. M. Auditory-somatosensory multisensory interactions in front and rear space. Neuropsychologia45, 1869-1877, doi:10.1016/j.neuropsychologia.2006.12.004 (2007).

23 Kitagawa, N. \& Spence, C. Audiotactile multisensory interactions in human information processing. Jpn. Psychol. Res.48, 158-173, doi:10.1111./j.1468-5884.2006.00317.x (2006).

24 Honda, S. et al. Proximal binaural sound can induce subjective frisson. Front. Psychol.11, 316, doi:10.3389/fpsyg.2020.00316 (2020).

25 Kitagawa, N. \& Igarashi, Y. Tickle sensation induced by hearing a sound. Jpn. J. Psychono. Sci.24, 121122 (2005).

26 Michael, G. A. \& Naveteur, J. The tickly homunculus and the origins of spontaneous sensations arising on the hands. Conscious. Cogn.20, 603-617, doi:10.1016/j.concog.2010.11.013 (2011). 
27 Michael, G. A. et al. Interacting effects of vision and attention in perceiving spontaneous sensations arising on the hands. Exp. Brain Res.216, 21-34, doi:10.1007/s00221-011-2901-y (2012).

28 Beissner, F. et al. Placebo-induced somatic sensations: a multi-modal study of three different placebo interventions. PLoS ONE10, e0124808, doi:10.1371/journal.pone.0124808 (2015).

29 Tada, K., Hasegawa, R. \& Kondo, H. M. Sensitivity to everyday sounds: ASMR, misophonia, and autistic traits. (under revision).

30 Violant-Holz, V. et al. Psychological health and physical activity levels during the COVID-19 pandemic: a systematic review. International journal of environmental research and public health17, 9419, doi:10.3390/ijerph17249419 (2020).

31 Blood, A. J. \& Zatorre, R. J. Intensely pleasurable responses to music correlate with activity in brain regions implicated in reward and emotion. Proc. Natl. Acad. Sci. U. S. A.98, 11818-11823, doi:10.1073/pnas.191355898 (2001).

32 Blood, A. J., Zatorre, R. J., Bermudez, P. \& Evans, A. C. Emotional responses to pleasant and unpleasant music correlate with activity in paralimbic brain regions. Nat. Neurosci.2, 382-387, doi:10.1038/7299 (1999).

33 Rickard, N. S. Intense emotional responses to music: a test of the physiological arousal hypothesis. Psychol. Music32, 371-388, doi:10.1177/0305735604046096 (2004).

34 Kumar, S. et al. The brain basis for misophonia. Curr. Biol.27, 527-533, doi:10.1016/j.cub.2016.12.048 (2017).

35 McGeoch, P. D. \& Rouw, R. How everyday sounds can trigger strong emotions: ASMR, misophonia and the feeling of wellbeing. Bioessays42, e2000099, doi:10.1002/bies.202000099 (2020).

36 Huron, D. Sweet Anticipation: Music and the Psychology of Expectation. (MIT Press, 2006).

37 Konečni, V. J. The aesthetic trinity: awe, being moved, thrills. Bulletin of Psychology and the Arts5, 2744, doi:10.1037/e674862010-005 (2005).

38 Heuchert, J. P. \& McNair, D. M. The Profile of Mood States 2nd Edition (POMS 2). (Multi-Health Systems, 2012).

\section{Figures}




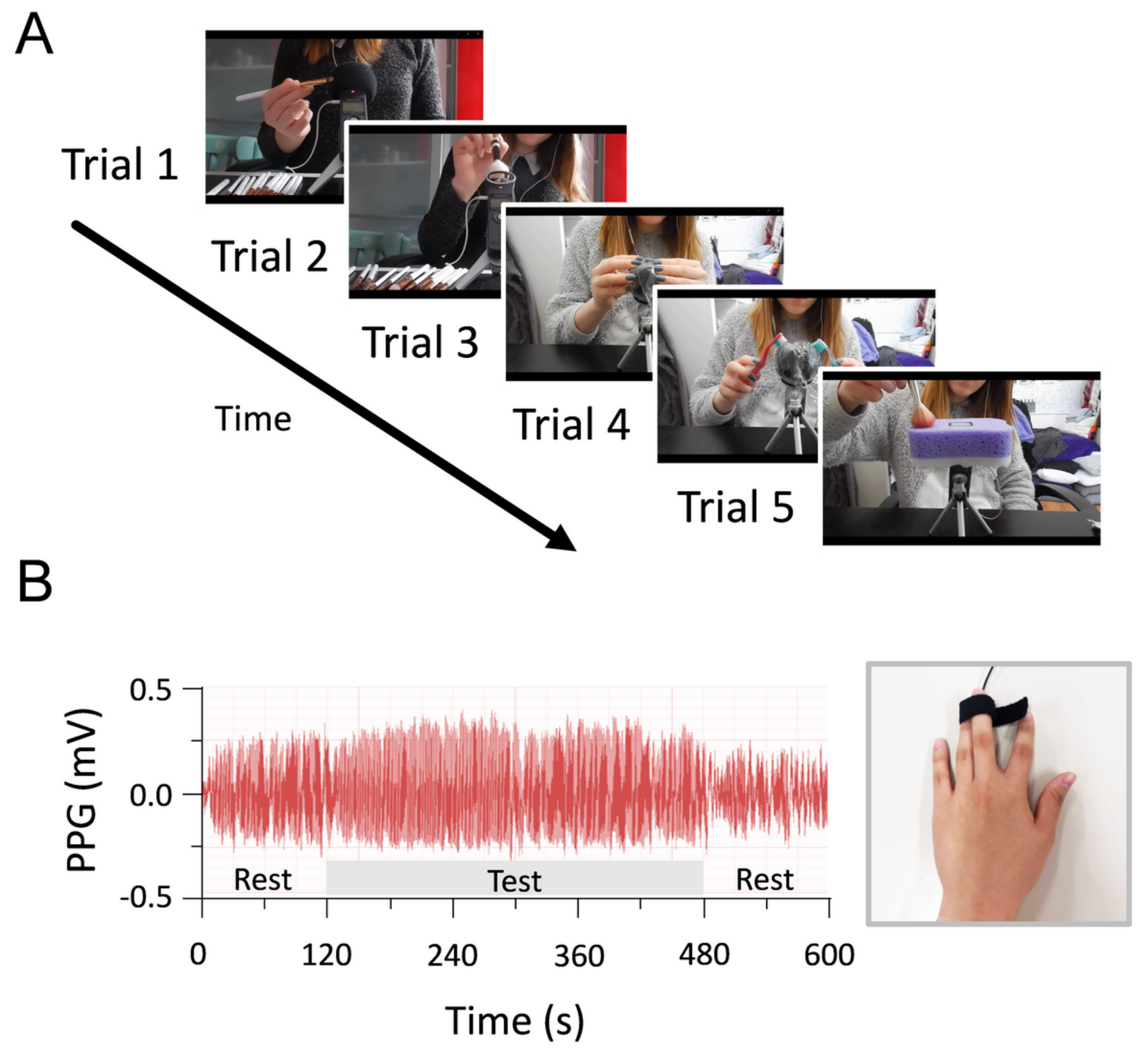

Figure 1

Task paradigm and photoplethysmography (PPG) data. (A) Experiment 1 consisted of audiovisual and audio-only conditions (five 2-min trials for each). Participants were randomly assigned to either condition. Under audiovisual conditions, they observed audiovisual stimuli and continuously indicated the subjective intensity of the ASMR using a 4-point Likert scale. Stimuli and task procedures under audioonly conditions were identical to those under audiovisual conditions, except for removal of visual information from stimuli. (B) Experiment 2 involved 6-min test periods and 4-min rest periods. PPG data were obtained from a sensor attached to a participant's left middle finger. Parasympathetic activation triggers vasodilation, resulting in high PPG amplitude. 
A
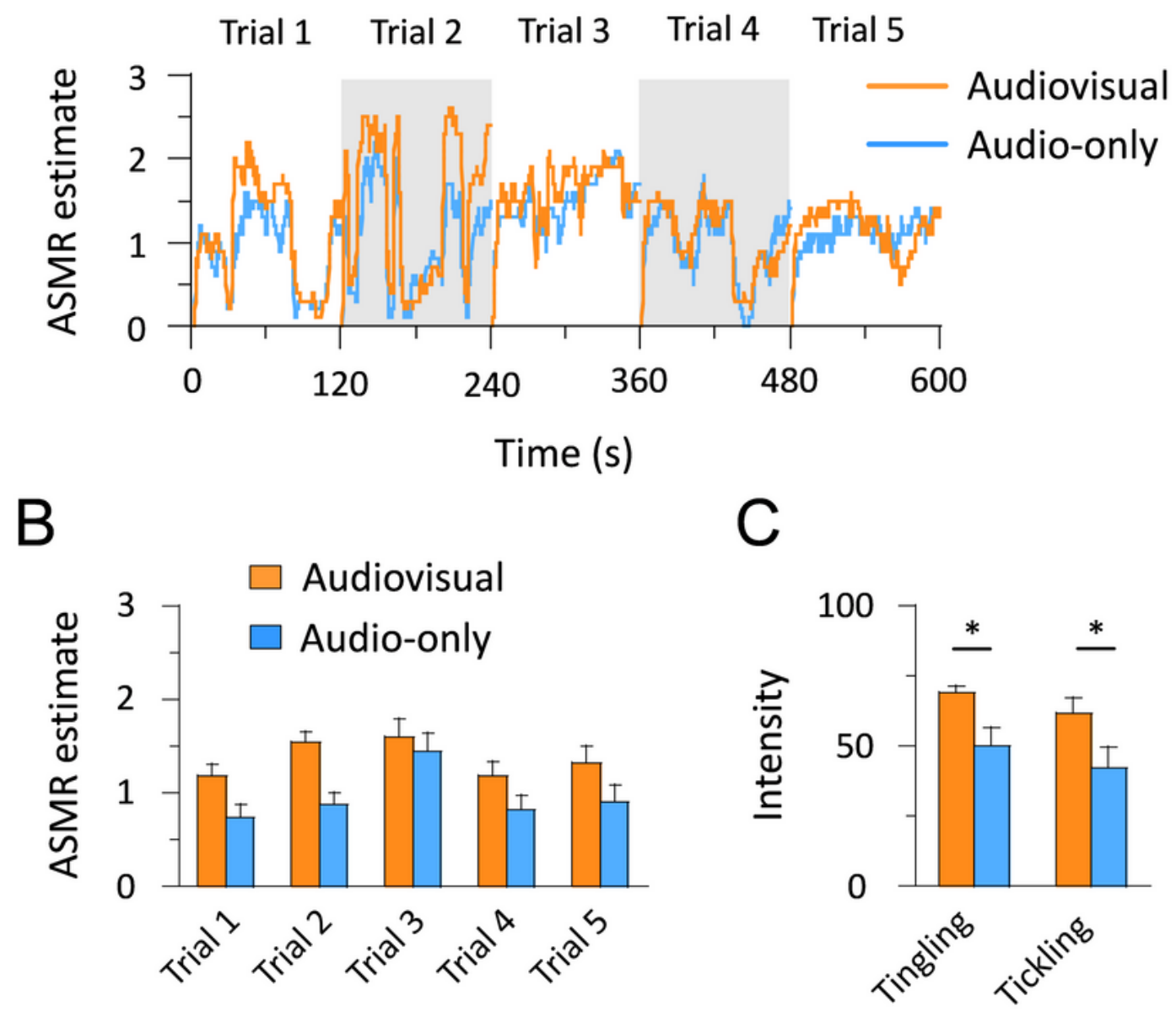

D

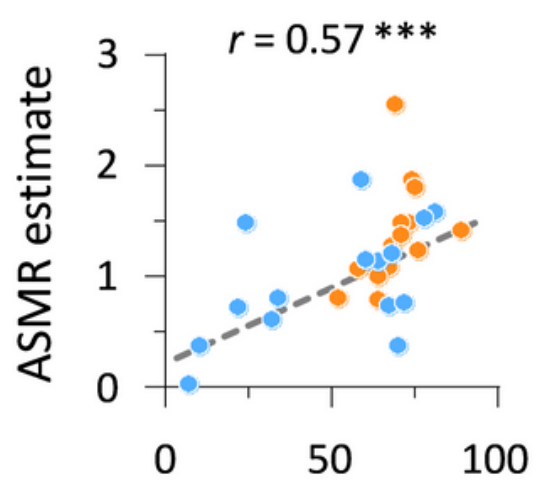

Tingling

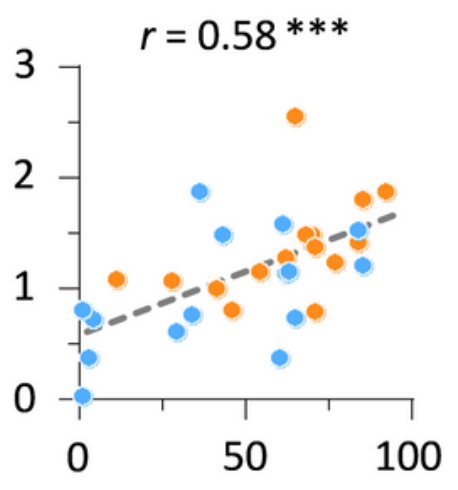

Tickling

Figure 2

Results of Experiment $1(\mathrm{~N}=30)$. (A) Time-series data of ASMR estimates averaged over participants under audiovisual and audio-only conditions ( $n=15$ for each). (B) Mean ASMR estimate for each 2-min trial. Estimates for all trials were greater under audiovisual conditions than under audio-only conditions. Error bars indicate standard errors of means. (C) Subjective ratings of sensations. Mean intensity of tingling and tickling sensations was greater under audiovisual conditions than under audio-only 
conditions. (D) Scatter plots for the relationship between ASMR estimates and subjective ratings. Dashed lines represent linear regression. ${ }^{* *} p<0.001,{ }^{*} p<0.05$.
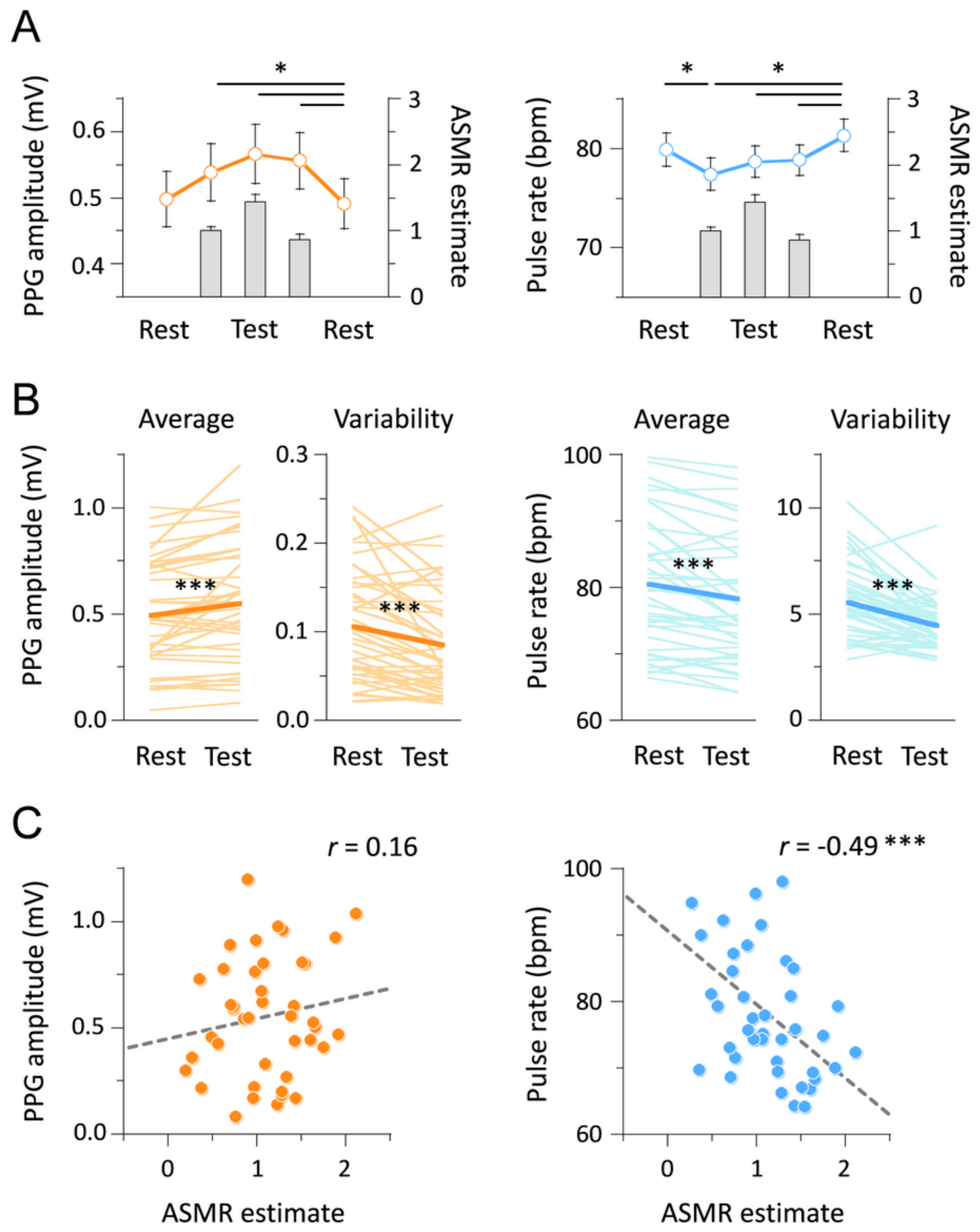

Figure 3

Results of Experiment $2(\mathrm{~N}=42)$. (A) Comparison of pulse wave amplitude (orange) and pulse rate (blue) between test and rest periods. Bars represent averaged ASMR estimates with standard errors of means. (B) Individual data of pulse wave amplitude and pulse rate. Thick lines indicate means of the measures. 
(C) The relationship between the ASMR estimate, pulse wave amplitude, and pulse rate. ${ }^{\star \star \star} \mathrm{p}<0.001$, ${ }^{\star} \mathrm{p}<$ 0.05 (Bonferroni correction for multiple comparisons).

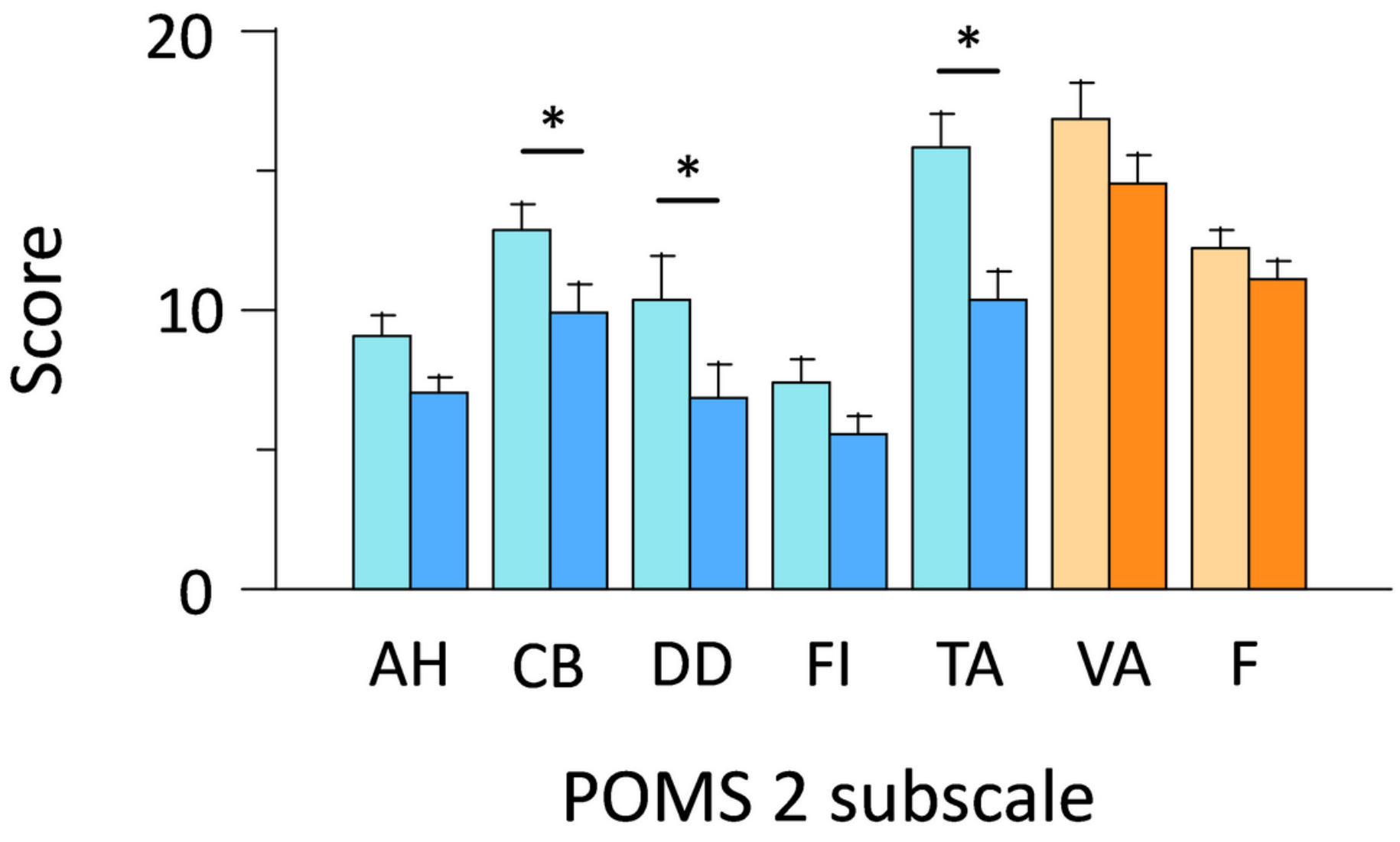

Figure 4

Results of Profile of Mood States 2nd Edition (POMS 2). AH (Anger-Hostility), CB (ConfusionBewilderment, DD (Depression-Dejection), FI (Fatigue-Inertia), and TA (Tension-Anxiety) subscales indicate negative mood (blue), whereas VA (Vigor-Activity) and F (Friendliness) subscales represent positive mood (orange). Means of all POMS 2 subscales decreased after the experiment (right bars) compared to before (left bars). Error bars indicate standard errors of means. ${ }^{*} \mathrm{p}<0.05$ (Bonferroni correction for multiple comparisons). 